\title{
Implementing quality improvement programs for colonoscopy: the emerging need for an international, standardized, multidisciplinary approach
}

Authors

Institutions
Matthew D. Rutter ${ }^{1,2}$, losif Beintaris ${ }^{1}$

${ }^{1}$ University Hospital of NorthTees - Gastroenterology, Stockton on Tees, Cleveland, United Kingdom

${ }^{2}$ Durham University - Medicine, Durham, United Kingdom submitted

10. December 2015

accepted after revision

21. December 2015

\section{Bibliography}

DOI http://dx.doi.org/

10.1055/s-0042-100278

Endoscopy International Open 2016; 04: E134-E135

(c) Georg Thieme Verlag KG

Stuttgart · New York

E-ISSN 2196-9736

\section{Corresponding author}

Matthew D. Rutter, MBBS, MD, FRCP

University Hospital of NorthTees - Gastroenterology

Stockton on Tees

Cleveland

United Kingdom

Phone: +44-1642-624557

Fax: +44-1642-383289

matt.rutter@nth.nhs.uk

\section{License terms}

(ब)(1) $\Theta \circledast$
The importance of quality in everyday endoscopy practice is increasingly recognized. The advent of organized bowel cancer screening programs has brought a focus on high-quality colonoscopy worldwide and has driven the desire to extend quality assurance to all aspects of endoscopic practice. Quality assurance has been shown to improve performance [1], leading to increased patient satisfaction and improved health outcomes [2].

A significant variation exists in the performance of endoscopists and of endoscopy units [3]. In colonoscopy, rates of cecal intubation, adenoma detection, and post-colonoscopy colorectal cancer (an indicator of overlooked or incompletely excised premalignant or malignant lesions) vary considerably between units [4], raising concerns about the quality of some services. These differences only come to light when performance is measured, without which there is no opportunity to support individuals or for endoscopy services to improve.

To maximize the potential to improve endoscopy quality, all domains of the service should be measured, including equipment, staffing levels, timeliness, adherence to clinical guidelines, and adherence to minimum recognized clinical quality standards of procedures.

However, establishing a quality assurance structure for endoscopists and endoscopy units is not a simple task. Candas and colleagues attempt to address this hot topic in this issue of EIO [5]. The authors performed a robust mixed-methods systematic review of current literature aiming to identify barriers and facilitators of colonoscopy quality program implementation in endoscopy units. They examined the issue from four different perspectives: endoscopists, nurses, patients, and healthcare managers. In total, 15 studies from across the globe were included in the analysis, most being questionnaires or surveys. Disappointingly, but perhaps not surprisingly, very few studies examined the issue from the perspective of patients, nurses or managers.

Mixed-methods review methodology, although technically challenging due to the methodological diversities between qualitative and quantitative studies [6] , is appealing as it incorporates qualitative and quantitative components of the subject under research and can potentially provide robust answers to complex questions. Candas and colleagues should be credited for selecting this method to determine factors that can facilitate policy changes in endoscopy units toward the goal of quality improvement. They grouped responses into three broad categories: features of continuous quality improvement (CQI) programs, attitudes and perceptions, and organizational characteristics.

A couple of themes emerge. First, receptive users understand and are willing to embrace quality improvement, particularly when there is clear evidence of gain and they have some sense of ownership of the process. Nevertheless, they desire such a process to be formative - involving education and training - and supportive rather than punitive. We concur that such a holistic approach is far more likely to succeed. Users express concern about the safeguarding of endoscopists' confidentiality: this is understandable and should be acknowledged when programmes are being instigated-in our experience using a degree of data anonymity gives individuals greater confidence that the process is supportive. The paper identifies that users want quality improvement programs to be voluntary; surprisingly the authors appear to support this. We would argue to the contrary: while a voluntary program is better than nothing, when possible, quality assurance programs should be mandatory, otherwise those whose performance is suboptimal may simply not participate, to the ongoing detriment of patient care. 
Second, users correctly identify that for quality improvement to work, it needs to be part of a broader culture of clinical excellence, incorporating strong leadership and multidisciplinary teamwork. For such a program to succeed in the long term, it needs to be organized and embedded in the routine activities of an endoscopy service. Management buy-in and support with adequate resourcing (both financial and time) is seen as important, as is keeping the scope of the project feasible by focusing on a manageable number of key performance measures. Their study also shows that there is a need to increase multidisciplinary involvement (nurse, managers, and patients, as well as clinicians) in the development of quality improvement programs and in their evaluation. This is especially pertinent for performance measures assessing the quality of pre- and post-endoscopy components of a service, including patient satisfaction and procedure timeliness.

While the paper does not deliver any surprises, it does nicely encapsulate the perceived challenges of implementing quality assurance programs, which may promote the success and endurance of future quality initiatives. Although the paper in this issue focuses on colonoscopy, the message it delivers is equally applicable to all endoscopy.

There are several aspects of quality improvement and performance measures that are not covered by the paper, presumably because they were not identified in the underlying studies. These include using objective performance measures less susceptible to "gaming," and using standardized, evidence-based performance measures to allow meaningful comparison between endoscopy services. We believe that these aspects are important. The ESGE, supported by UEG, is currently developing such performance measures for endoscopy, incorporating endoscopy service measures along with lower gastrointestinal, upper gastrointestinal, small bowel, and pancreatobiliary endoscopy. This process is truly multidisciplinary, involving clinicians, nurses, managers and, importantly, patients [7].

The need for a standardized, global approach to quality improvement processes is now more relevant than ever. Comparison of local quality data with standardized, international performance measures can be a powerful motivation for individual services to aim to higher standards, reducing performance variation between operators and units. That, of course, is not an easy task, as it requires commitment from all involved parties, including management. Nevertheless, the goal of improved patient health outcomes alone justifies coordinated efforts towards this approach.

\section{Competing interests: None}

\section{References}

1 Gavin DR, Valori RM, Anderson JT et al. The national colonoscopy audit: a nationwide assessment of the quality and safety of colonoscopy in the UK. Gut 2013; 62: $242-249$

2 Rees CJ, Rajasekhar PT, Rutter MD et al. Quality in colonoscopy: European perspectives and practice. Expert review of gastroenterology \& hepatology 2014; 8: 29-47

3 Chen SC, Rex DK. Endoscopist can be more powerful than age and male gender in predicting adenoma detection at colonoscopy. The American journal of gastroenterology 2007; 102: 856-861

4 Valori R, Morris E, Thomas J et al. OC-061 Rates Of post colonoscopy colorectal cancer (pccrc) are significantly affected by methodology, but are nevertheless declining in the Nhs. Gut 2014; 63: A30

5 Candas B, Jobin G, Dubé C et al. Barriers and facilitators to implementing continuous quality improvement programs in colonoscopy services: A mixed methods systematic review. Endosc Int Open 2016; 4: $118-133$

6 Heyvaert $M$, Maes $B$, Onghena P. Mixed methods research synthesis: definition, framework, and potential. Qual Quant 2013; 47: 659-676

7 Rutter MD, Senore C, Bisschops R et al. The European Society of Gastrointestinal Endoscopy Quality Improvement Initiative: developing performance measures. Endoscopy 2016; 48: 81 -89 